Editorial

\title{
Nuevos desafíos en Tecnologías de Información y Comunicación
}

\section{New challenges in Information and Communication Technologies}

\author{
Jezreel Mejía ${ }^{1}$, Mirna Muñoz ${ }^{1}$ Giner Alor-Hernández², Claude Y Laporte ${ }^{3}$ \\ \{jmejia, mirna.munoz\}@cimat.mx; galorh@orizaba.tecnm.mx; claude.laporte@etsmtl.ca
}

${ }^{1}$ Centro de Investigación en Matemáticas- Unidad zacatecas, Parque Quantum, Ciudad del Conocimiento, Avenida Lasec, andador Galileo Galilei, manzana 3 lote 7, C. P. 98160, Zacatecas, México.

${ }^{2}$ División de Estudios de Postgrado e Investigación, Instituto Tecnológico de Orizaba, Avenida Oriente 9 No. 852 Col. Emiliano Zapata, 94330, Orizaba, Veracruz, Orizaba, México, Orizaba, Veracruz, México.

${ }^{3}$ Department of Software and IT Engineering, École de technologie supérieure, Montreal, Canada

DOI: 10.17013/risti.41.o

\section{Introducción}

Los artículos presentados en este número fueron seleccionados de los artículos aceptados en el Noveno Congreso Internacional de Mejora de Procesos Software (CIMPS) 2020, con sede local en la Ciudad de Mazatlán, Sinaloa, México del 21 al 23 de octubre del 2020. Este congreso ha tenido una tasa de aceptación del 30\% de los artículos enviados.

Los artículos publicados en este número de la Revista Ibérica de Sistemas y Tecnologías de la Información (RISTI), aborda temas relacionados a "Nuevos Desafíos en Tecnologías de Información y Comunicación”. Los artículos presentan versiones extendidas y mejoradas de las versiones originales que han sido presentados en el congreso CIMPS 2020.

\section{Estructura}

En el primer artículo, los autores abordan el uso de Kanban, ya que se ha popularizado e incrementado en la industria del software. Sin embargo, la falta de experiencia y la difícil 
formación de los nuevos practicantes limitan su adopción dentro de las organizaciones. Una alternativa para resolver este problema, es hacer uso de juegos serios como herramienta en la capacitación del capital humano, ya que impulsan un cambio en su actitud, motivación y compromiso. Los autores presentan una biblioteca de juegos serios para enseñar Kanban desarrollada con el propósito de ayudar a los entrenadores a seleccionar el juego serio que cumpla con sus objetivos de enseñanza. La biblioteca fue validada por un grupo de expertos en Kanban.

En el segundo artículo presenta una plataforma virtual de aprendizaje gamificada donde los alumnos se diviertan aprendiendo a programar influyendo positivamente en su motivación por el desarrollo de actividades autónomas de aprendizaje, además que también permitió mejorar su rendimiento académico. Esta propuesta se aplicó en la asignatura Programación I (P-I) de la Facultad de Ingeniería y Ciencias Aplicadas (FICA) de la Universidad Central de Ecuador (UCE). Esta plataforma aplica estrategias de gamificación implementadas en una plataforma virtual de aprendizaje como apoyo a clases presenciales. Jefferson

El tercer artículo, se centra en evaluar el desempeño académico de los estudiantes de la Licenciatura en Gastronomía de la Universidad Autónoma de Sinaloa (UAS) en entornos virtuales, específicamente en la asignatura de estadística. La metodología aplicada fue cuasi-experimental con un enfoque longitudinal descriptivo mixto por encuesta. Además, se aplicó el modelo de Progrogramación Neurolingística (PNL) para conocer el estilo de aprendizaje predominante en los estudiantes. Finalmente, analizá el desempeño académico de los estudiantes durante el desarrollo de dos unidades de aprendizaje.

El cuarto artículo presenta una herramienta también llamada ambiente de desarrollo en conjunto de un lenguaje de programación, en idioma español, que permita programar la generación de patrones geométricos, como un apoyo a la enseñanza de STEM en México. El objetivo de la herramienta pretende que el aprendizaje de la programación se mas fácil de lo que habitualmente se percibe.

El quinto artículo presenta los factores clave para la adopción de dos tecnologías inteligentes: Big Data y el Internet de las Cosas en las universidades. Esta caracterización e integración permite concluir sobre la necesidad de alineación de la tecnología con los procesos de la organización, exigiendo una mayor interacción con la alta dirección, debido a que una universidad inteligente se centra en la mejora de su infraestructura tecnológica para alcanzar sus objetivos de calidad educativa.

El sexto artículo propone una herramienta que apoye a las Mipymes a evaluar los procesos y el proyecto con base a la norma ISO/IEC 29110 para presentarse en una futura certificación. Para ello, la herramienta fue implementada en una Mipyme de desarrollo de software previo a iniciar un proceso de certificación en esta norma. Después de la implementación de esta herramienta, la Mipyme obtuvo la certificación en la norma ISO/IEC 29110 de una manera mas controlada y guiada, ya que le permitió solucionar las observaciones con antelación antes de presentarse a un proceso de certificación.

Con respecto al séptimo artículo presenta un marco de trabajo desarrollado para aplicación de tecnología Blockchain en el ámbito de la educación. El marco está compuesto por un conjunto de pasos como referencia para elaborar aplicaciones 
descentralizadas (DApps), además, presenta la elección de herramientas utilizadas en diferentes propuestas de este ámbito. Por último, se desarrolla una arquitectura robusta que puede ser implementada en diferentes DApps. El marco se valida en el caso de certificación académica y la gestión de propiedad intelectual, que incluye beneficiados y un análisis del aporte de esta tecnología en cada ámbito

Finalmente, el octavo artículo aborda uno de los grandes problemas de la humanidad que es la excesiva cantidad de residuos que se generan a nivel mundial; Aproximadamente, el 50\% de los residuos generados en las ciudades son orgánicos, para darles un valor agregado se pueden transformar en composta. Por lo tanto, este articulo, presenta el desarrollo de un software embebido para un compostero doméstico inteligente. En este trabajo se presentan los resultados de la primera iteración del proceso de desarrollo de software utilizado.

\section{Agradecimientos}

Agradecemos al director de la Revista Álvaro Rocha por otorgar la oportunidad para dar a conocer los trabajos mas relevantes presentados en el congreso CIMPS 2020. Asimismo, expresamos nuestra gratitud a los autores de los trabajos publicados, lo cual hizo posible este número en RISTI. Damos la enhorabuena a todos aquellos autores cuyos artículos han sido publicados en este número de RISTI. 